\title{
Imaging Atomic Dynamics in 2D Silica Glass with Low-Voltage Aberration- Corrected TEM
}

Pinshane Y. Huang ${ }^{1}$, Jonathan S. Alden ${ }^{1}$, Simon Kurasch ${ }^{2}$, Ashivni Shekhawat ${ }^{3}$, Alexander A. Alemi ${ }^{3}$, James P. Sethna ${ }^{3}$, Ute Kaiser ${ }^{2}$, and David A. Muller ${ }^{1,7}$

1. School of Applied and Engineering Physics, Cornell University, Ithaca, NY, USA 14853

2. Electron Microscopy Group of Materials Science, University of Ulm, Ulm, Germany 89081

3. Laboratory of Atomic and Solid State Physics, Cornell University, Ithaca, NY, USA 14853

4. Kavli Institute at Cornell for Nanoscale Science, Cornell University, Ithaca, NY, USA 14853

Tracking the dynamics of individual atoms in glassy and disordered solids has been considered difficult, if not impossible. Yet, characterizing atomic motion is critical for understanding a wide range of behavior in amorphous materials - particularly the mechanisms of plastic deformation. Here, we demonstrate a new approach to this problem: applying low-voltage $(80 \mathrm{kV})$ aberration-corrected transmission electron microscopy (TEM) as a tool to excite and image dynamics in ultra-thin glasseshere a two-dimensional (2D) silica glass[1,2]. This combination of carefully chosen beam voltage and novel 2D materials makes it possible to observe and track atomic motion in an amorphous material for the first time. The resulting data include surprisingly rich and beautiful videos of elastic and plastic deformations in a 2D glass, providing insight to the atomic-scale mechanisms for plastic deformation in amorphous solids, which unlike in crystals, are not well understood. Moreover, our examples - of bond exchange, plastic shear deformation, and rearrangements at a solid-liquid interface-illustrate the wideranging materials physics that can be now studied at atomic-resolution via TEM imaging of 2D glasses.

Combining 2D glasses and low-voltage TEM is a powerful tool for imaging atom motion and quantifying strain on the atomic scale. Amorphous 2D silica is a layered polymorph of $\mathrm{SiO}_{2}$; its $2 \mathrm{D}$ nature makes it the first glass that can be imaged at atomic resolution[1, 2]. Figure 1a-d, f show atomicresolution TEM images of glassy $2 \mathrm{D}$ silica. The darkest spots in the images represent the location of two stacked $\left(\mathrm{SiO}_{4}\right)^{4-}$ tetrahedra. The $80 \mathrm{kV}$ beam energy is above the thresholds for knock-on and ionization damage in $\mathrm{SiO}_{2}$. Prolonged e-beam irradiation produces mobile vacancies, enabling plastic deformation, flow, and even local melting well below the glass transition temperature[3, 4]. These beam-induced transformations provide an avenue to observe and study materials phenomena that would typically occur at high stresses and temperatures. An example is shown in Figure 1a-d: after 14 images (28 s) and a total dose of $\sim 50$ electrons $/ \mathrm{nm}^{2}$, the 5-7-5-7 cluster transforms into a 6-6-6-6 cluster through the rotation of one bond. Importantly, because the probabilities for atom displacements are low compared to the dose required for imaging, our $\sim 1-2 \mathrm{~s}$ frame rates are sufficient to capture the elastic and plastic deformation of these atoms. We track the position of each atom over time during this rearrangement, producing the trajectory map in Figure 1e and larger-area displacement map in Figure $1 \mathrm{f}-\mathrm{g}$.

We observe clear correlations between the single bond rearrangement and the elastic deformations of the surrounding sheet. We quantify this elastic deformation by plotting the $2 \mathrm{D}$ strain tensor $\varepsilon$ and the local rotation matrix $\boldsymbol{\omega}$ in Figure 2a-d[5]. The four-lobed, quadrupole-like signal in $\boldsymbol{\omega}$ is strong and extended when compared to the components of $\varepsilon$. These calculations confirm that the nanometer-scale, "longrange" elastic behavior of the material near the bond rearrangement is dominated by local rotation rather than by strain. These results indicate a promising new avenue for studying rearrangement in glasses. 


\section{References:}

[1] P. Y. Huang, et al., Nano Letters 12 (2), 1081-1086 (2012).

[2] L. Lichtenstein, et al., Angew. Chem. 51 (2), 404-407 (2012).

[3] K. Zheng, et al., Nature Communications 1 (3), 1-8 (2010).

[4] L. W. Hobbs and M. R. Pascucci, Le Journal de Physique Colloques 41 (C6), 237-242 (1980).

[5] L. D. Landau and E. M. Lifshitz, Theory of Elasticity (Pergamon Press, New York, 1970).

[6] This work was supported by the NSF through the Cornell Center for Materials Research (NSF DMR-1120296) and the National Science Foundation Graduate Research Fellowship under Grant No. DGE-0707428, as well as by the German Research Foundation (DFG), the Ministry of Science, and the Arts Baden-Württemberg within the SALVE (Sub-Angstrom Low-Voltage Electron microscopy) project.

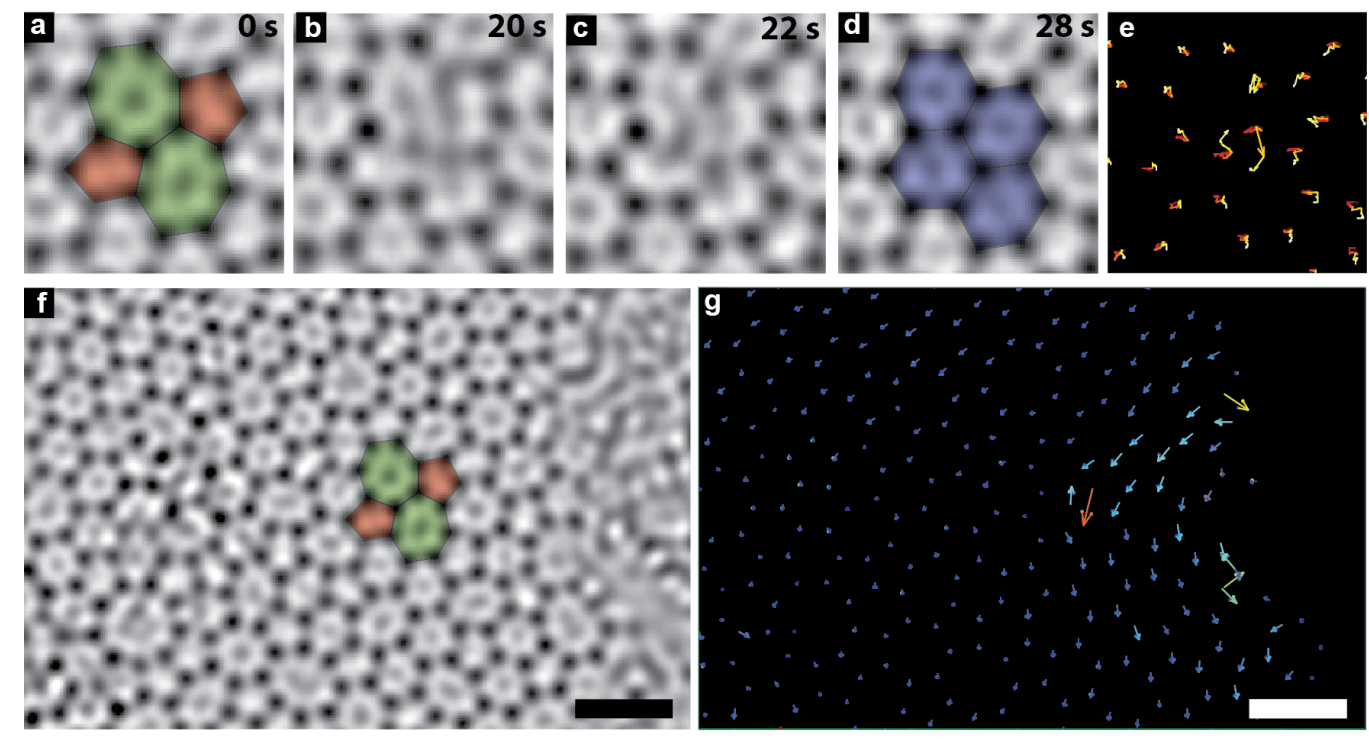

Figure 1. Imaging bond exchange and corresponding atomic displacements in a glassy 2D sheet. a-d) TEM image series of amorphous 2D silica. A bond rotation transforms a 5-7-5-7 cluster into a 6-6-6-6 cluster during a video spanning 14 images $(28 \mathrm{~s})$ and a dose of $\sim 50$ electrons $/ \mathrm{nm}^{2}$. The dark spots are SiO-Si columns; each dark spot represents one atomic site. e) A trajectory map of atomic sites from frame to frame. Color (red to yellow) indicates time of displacement. f) Larger-area image of 2D glassy structure in (a) and g) corresponding first-to-last frame displacement map. Scale bars $2 \mathrm{~nm}$.

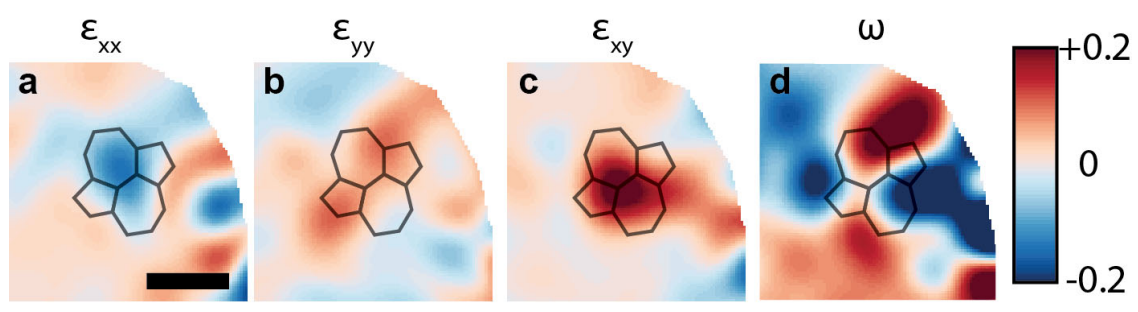

Figure 2. Strain analysis of 5-7-5-7 to 6-6-6-6 bond exchange. a-d) Experimental strain components $\varepsilon_{\mathrm{xx}}$, $\varepsilon_{\mathrm{yy}}$, and $\varepsilon_{\mathrm{xy}}(\mathrm{a}-\mathrm{c})$ and local rotation $\omega(\mathrm{d})$ due to the bond exchange in Figure 1. These are calculated by comparing the change in position of atoms between the first and last image frames, excluding the two atoms at the center of the bond exchange to highlight only elastic behavior. The strain field is dominated by a strong four-lobed, quadrupole-like signal in $\omega$. Scale bar $1 \mathrm{~nm}$. 